\title{
Correction to: The LRE Map: what does it tell us about the last decade of our field?
}

\author{
Riccardo Del Gratta ${ }^{1}$ (D) Sara Goggi ${ }^{1}$. \\ Gabriella Pardelli ${ }^{1} \cdot$ Nicoletta Calzolari $^{1}$
}

Published online: 16 February 2021

(C) Springer Nature B.V. 2021

\section{Correction to: Lang Resources \& Evaluation https://doi.org/10.1007/s10579-020-09520-6}

In the original publication of the article the "Abstract" section was published with an error. The corrected version of the Abstract is given below:

\begin{abstract}
The LRE Map of Language Resources was introduced at LREC 2010. Its intended purpose was: "to shed light on the vast amount of resources that represent the background of the research presented at LREC". It also aimed at a change of culture in the field, actively engaging each researcher both in the documentation task about resources and in sharing resources. When we started to use it regularly also in other conferences, it became clear that it was an innovative instrument able to provide a
\end{abstract}

The original article can be found online at https://doi.org/10.1007/s10579-020-09520-6.

Riccardo Del Gratta

riccardo.delgratta@ilc.cnr.it

Sara Goggi

Sara.Goggi@ilc.cnr.it

Gabriella Pardelli

Gabriella.Pardelli@ilc.cnr.it

Nicoletta Calzolari

Nicoletta.Calzolari@ilc.cnr.it

1 Istituto di Linguistica Computazionale “A. Zampolli”, CNR, Via Moruzzi 1, 56124 Pisa, Italy 
picture of the field and its evolution as reflected by the creation and use of Language Resources. After 9 years we revisit the Map, considerably extending the data analysed in an LREC 2018 paper. The LRE Map data analysed here have been provided by the authors of 21 conferences during the phase of submission of papers, and contain information about 9405 resources. We analyse the LRE Map data from many different viewpoints and the paper reports on the global picture, along the many Map dimensions, on different trends emerging from a diachronic perspective and finally on some comparisons between five editions of the two major conferences present in the Map: LREC and COLING.

The original article has been corrected.

Publisher's Note Springer Nature remains neutral with regard to jurisdictional claims in published maps and institutional affiliations. 